
\title{
3 Research Square \\ Burn the Base to Get the Polyp Erased: A Novel Technique to Reduce Post-EMR Recurrence
}

John Maynard Levenick ( $\nabla$ jlevenick@hmc.psu.edu )

Penn State College of Medicine https://orcid.org/0000-0001-6203-4650

Adnrew J Groff

University of Pittsburgh Medical Center

Carl Manzo

Penn State Health Milton S. Hershey Medical Center

Courtney Lester

Penn State Health Milton S. Hershey Medical Center

Jennifer L Maranki

PSHMC: Penn State Health Milton S Hershey Medical Center

\section{Research article}

Keywords: endoscopic mucosal resection, colon polyps, Hybrid APC

Posted Date: January 19th, 2021

DOl: https://doi.org/10.21203/rs.3.rs-148499/v1

License: (c) (1) This work is licensed under a Creative Commons Attribution 4.0 International License. Read Full License 


\section{Abstract}

Background and Aims: Endoscopic mucosal resection (EMR) has become the standard for removing large colon polyps but has a 10-30\% recurrence rate using standard techniques. Data shows improved recurrence rates with focal therapy of the edge of the resection base using cautery. We examine a novel technique, hybrid APC assisted EMR, which treats both the edge and the base with cautery to assess its effect on local recurrence.

Methods: We reviewed all EMRs of polyps $>2 \mathrm{~cm}$ by a single endoscopist with six month follow-up from May 2018-November 2019 using both standard EMR as well as hybrid APC assisted EMR to assess local recurrence as well complications.

Results: 48 patients with 59 polyps removed by EMR had full six month follow up with a mean age of 66.1 years of age, $45 \%$ were female. 30 polyps were removed by hybrid APC assisted EMR and 29 removed with standard EMR. Overall, $0(0 \%)$ polyps in the h-APC arm had local recurrence while $6(20.7 \%)$ in the standard group had histological proven local recurrence $(p=0.01)$. Post-resection bleeding occurred in 6 patients, 2 in the hAPC arm and 4 in the standard arm $(p=0.41)$. Conclusions: Hybrid APC assisted EMR was superior to conventional EMR for local recurrence after removal of large colon polyps and trended towards a less post-EMR bleeds.

\section{Background}

Lateral spreading lesions (LSLs) are large, flat, sessile polyps with pre-malignant potential. Out of all polyps removed during colonoscopy, $5 \%$ are LSLs greater than $10 \mathrm{~mm}$ in size. ${ }^{1}$ LSLs greater than $20 \mathrm{~mm}$ in size are considered high-risk lesions with even greater chance of becoming malignant over time. Endoscopic mucosal resection (EMR) has proven to be a safe and effective surgery-sparing technique for removal of LSLs. ${ }^{2}$ Compared to surgical resection, EMR is cost effective, allows for rapid recovery, and decreases adverse events with significant improvement in morbidity and mortality. ${ }^{3-5}$

The fundamental technique for EMR was developed in 1955 when Rosenberg created a submucosal saline injection to improve safety of applying thermal therapy in the form of fulguration for treatment of polyp tissue. ${ }^{6}$ This same technique was later used in the 1970s for creating a submucosal injection to lift flat lesions from the mucosal surface to improve polypectomy of these lesions. ${ }^{7}$ In the 1990s, the technique was further refined and started to become standard therapy for resections of LSLs in Japan. ${ }^{8}$

Although safer than surgery, adverse events occur at a known rate including perforation in $1-2 \%$ and delayed bleeding in 5-10\% of cases. ${ }^{9-10}$ However, a major concern with EMR continues to be local recurrence. ${ }^{11-12}$ Polyp recurrence at the resection site of EMR is quoted anywhere from $10-30 \% .^{13-17}$

EMR of LSLs is most often done in a piecemeal fashion. When this occurs, there may be adherent macroscopic disease which can be a cause of local recurrence. ${ }^{17}$ These areas of visible, residual 
neoplasia had previously been fulgurated first with argon plasma coagulation (APC) and more recently resected with tissue avulsion using hot or cold biopsy forceps. ${ }^{17-18}$ Local recurrence rates were exceptionally high at around $60 \%$ when APC alone was used on visible residual neoplasia and, though improved, remained elevated at roughly $10 \%$ with hot avulsion. ${ }^{17}$ Despite complete resection of macroscopic polyp tissue, there remained a high local recurrence rate causing endoscopists to surmise that microscopic residual tissue may be left behind at the edges of the resection bed. With this theory, Bourke et al investigated whether resection of 3-5 $\mathrm{mm}$ of macroscopically normal appearing mucosa around the EMR resection bed would reduce the rate of local recurrence. ${ }^{19}$ Surprisingly, they found essentially no difference in recurrence rates between standard EMR and wide-excision EMR. Most recently, local ablative therapy using snare-tip soft coagulation (STSC) around the edges of the resection site was shown to significantly decrease polyp recurrence rates from $21 \%$ in the control arm to $5 \%$ in those treated with STSC. ${ }^{20}$ Though this method did show an improvement in recurrence rates overall, an average of $5 \%$ of resections continued to show residual disease at the first surveillance colonoscopy with some endoscopists individually having up to $20 \%$ endoscopic recurrence and up to $30 \%$ histologic recurrence. The STSC technique was studied at another center who experienced a $12 \%$ recurrence rate at first surveillance colonoscopy which was an improvement from $30 \%$ recurrence in patients at the same center prior to incorporating this technique. ${ }^{21}$ We thereby hypothesize that recurrence not only originates from microscopic residual disease in the borders of the polypectomy site, but also within the base which to date has not been systematically treated.

We introduce the novel technique of hybrid APC (hAPC)-assisted EMR that allows for APC treatment of the borders and, perhaps most importantly, the base of the post-polypectomy site. With hAPC-assisted EMR, re-lifting the post-polypectomy submucosal bed with a needleless water jet allows for the safe application of thermal therapy to the resection bed to treat any residual microscopic disease. The aim of the present study was to assess polyp recurrence and outcomes in patients at a single center 6 months following treatment with hAPC-assisted EMR versus standard EMR by a single endoscopist.

\section{Methods}

\section{Patient Selection and Design}

This single-center, retrospective study analyzed all participants who underwent EMR by a single endoscopist for large colonic polyps (> $20 \mathrm{~mm}$ ) and completed a 6-month surveillance colonoscopy between May 2018 and November 2019. Participants were assigned to undergo standard EMR or hAPCassisted EMR based on the availability of the jet feature on the electrosurgery tower. The single jet in the endoscopy suite may be designated for use in other rooms with other providers for endoscopic mucosal dissection, ablation of dysplastic Barrett's esophagus, or per-oral endoscopic myotomy on certain days.

All patients between 18 and 89 years of age with a > $20 \mathrm{~mm}$ non-pedunculated colonic polyp were eligible for the study. Patients with biopsy-proven invasive carcinoma within a polyp, inflammatory bowel 
disease, poor general health (ASA Class >3), poor bowel preparation, or NICE Classification type III polyps were excluded from the study. This study was approved by the Penn State Milton S. Hershey Medical Center Institutional Review Board (IRB \#12499). All participants provided informed consent for the procedure.

\section{Procedure}

Prior to their colonoscopy, each patient underwent a standard, split-dose bowel preparation. Polyp characteristics including size, NBI International Colorectal Endoscopic (NICE) ${ }^{22}$ and Paris ${ }^{23}$ classifications, and granularity were noted and documented in the electronic endoscopy reporting system.

Following documentation of polyp characteristics, standard EMR was used for polypectomy. ${ }^{24} \mathrm{~A}$ submucosal injection containing normal saline and a contrast agent (ie methylene blue or indigo carmine) was used for lifting the polyp from the muscularis propria. The adequacy of the lift was carefully assessed. At that point, the polyp was removed in piecemeal fashion or en bloc using a hot snare with electrocautery (Erbe EndoCut). If macroscopic residual neoplastic tissue was present, all sites were removed with hot or cold avulsion. If more than one polyp was found that met the polyp inclusion criteria, it was counted separately and removed using the assignment strategy described above. Periprocedural antithrombotic medications were managed according to professional society guidelines. $^{25-26}$

For the intervention group, the ERBE ViO300D system was assembled. An injectate solution of was mixed as described above. Using the needleless waterjet of the Hybrid APC system, the lesion was lifted with an effect of 30-50 (Fig. 1). Once the lesion had been appropriately lifted and assessed, EMR was performed in typical fashion, as above. We performed all resection with the Endo Cut setting with an effect of 2, cut duration of 1 , and cut interval of 4 . After the neoplasia had been completely removed with or without avulsion, the base and borders were re-lifted with the needleless waterjet to create a heat sink in order to avoid deep mucosal injury during ablation. APC was then performed using a flow of $0.8 \mathrm{~L} / \mathrm{min}$ and 40 watts. The entire base of the site and the circumferential borders of the lesions undergo APC in their entirety. At the discretion of the endoscopist, endoscopic clips were used to close the post-polypectomy site.

\section{Outcomes}

The primary outcome measure was the rate of precancerous polyp recurrence at a 6-month surveillance colonoscopy. Polyp recurrence was defined as the presence of a precancerous polyp using histopathologic analysis at follow-up. If present, all macroscopic residual tissue was removed at the follow-up colonoscopy. If no macroscopic disease was found, biopsies were taken of the scar to ensure the absence of microscopic disease.

Secondary outcome measures were the rate of major adverse events including perforation, postpolypectomy bleeding, and post-polypectomy syndrome for 30 days following colonoscopy. Post- 
polypectomy bleeding was defined as a severe bleeding event which required hospitalization, blood transfusion, or intervention to stop the bleeding. Post-polypectomy syndrome was defined as patients presenting with fever, leukocytosis, and/or abdominal pain within one week of colonoscopy. These major adverse events were measured by phone calls, medical records, and/or follow-up clinic visit within 30 days following the colonoscopy.

\section{Statistical Analysis}

Means and frequencies were calculated to describe sample demographics, polyp characteristics, and procedural details as well as the incidence of polyp recurrence at the 6-month follow-up. T-tests and Fisher's exact tests were used to assess differences between groups in means and proportions, respectively. In addition, t-tests and Fisher's exact tests were used to determine whether there were differences in polyp or procedural variables between those with versus those without polyp recurrence at 6-months.

\section{Results}

A total of 48 patients with 59 polyps were included in this single-center study. The mean age of included patients was 66.1 years with $45.8 \%$ of patients being female. Baseline characteristics between the two groups were comparable. Thirty polyps (50.8\%) received hAPC-assisted EMR and the other 29 polyps (49.2\%) received standard EMR. The mean polyp size was $31.6 \mathrm{~mm}$ and the most common locations for the polyps were the cecum, transverse colon, and ascending colon, respectively. Mean polyp size and polyp location were comparable between groups; however, there was a significant difference in Paris Classification $(p=0.03)$. In the hAPC-assisted EMR group, the most common Paris Classifications were $\mathrm{Ila}$, Is, and Ila/Ilc. In the standard EMR group, the most common Paris Classifications were Ila, Ilb, and Is/lla (Table 1). 
Table 1

Polyp Characteristics

\begin{tabular}{|lllll|}
\hline & Overall $(\mathbf{n = 5 9 )}$ & hAPC $(\mathbf{n = 3 0})$ & No hAPC $(\mathbf{n = 2 9 )}$ & p-value \\
\hline Mean polyp size, in mm (SD) & $31.6(13.7)$ & $32.9(13.7)$ & $30.2(13.7)$ & 0.45 \\
\hline Location, $\mathrm{n}(\%)$ & $39(66.1)$ & $21(70.0)$ & $18(62.1)$ & 0.80 \\
Right colon & $11(18.6)$ & $5(16.7)$ & $6(20.7)$ & \\
Transverse colon & $9(15.3)$ & $4(13.3)$ & $5(17.2)$ & \\
Left colon & & & & \\
Paris Classification, $\mathrm{n}(\%)$ & $9(15.3)$ & $6(20.0)$ & $3(10.3)$ & \\
Is & $19(32.2)$ & $11(36.7)$ & $8(27.6)$ & \\
Ila & $8(13.6)$ & $0(0.0)$ & $8(27.6)$ & \\
Ilb & $13(22.0)$ & $7(23.3)$ & $6(20.7)$ & \\
Is/lla & $3(5.1)$ & $1(3.3)$ & $2(6.9)$ & \\
Ila/llb & $7(11.9)$ & $5(16.7)$ & $2(6.9)$ & \\
Ila/llc & & & & \\
\hline
\end{tabular}

There was a significant difference between the adequacy of the polyp lift between the hAPC-assisted EMR group compared to the standard EMR group $(p=0.03) .76 .7 \%$ of the patients undergoing hAPCassisted EMR had a complete polyp lift, compared to only $44.8 \%$ of patients in the standard EMR group. The histology of the polyps, the proportion of piecemeal resections, and the percentage of complete resection were similar between groups.

\section{Primary Outcome}

Polyp recurrence at the 6-month surveillance colonoscopy occurred in a total of 6 patients $(10.2 \%)$, all of whom were in the standard EMR group (20.7\%). No polyp recurrence was found in the hAPC- assisted EMR group. The difference between groups was statistically significant $(p=0.01)$ with higher recurrence in patients treated with standard EMR compared to hAPC-assisted EMR (Table 2). There were no statistically significant differences detected between those with versus those without polyp recurrence at 6-months in polyp size $(p=0.75)$, adequacy of lift $(p=0.26)$, polyp location $(p=0.56)$, piecemeal resection $(p=0.17)$, or histology $(p=0.23)$. 
Table 2

Polyp Outcomes

\begin{tabular}{|lllll|}
\hline & Overall $(\mathbf{n = 5 9 )}$ & $\begin{array}{l}\text { Hybrid APC } \\
(\mathbf{n = 3 0})\end{array}$ & $\begin{array}{l}\text { No Hybrid APC } \\
(\mathbf{n}=\mathbf{2 9})\end{array}$ & p-value \\
\hline Adequacy of Lift, $\mathbf{n}(\%)$ & $36(61.0)$ & $23(76.7)$ & $13(44.8)$ & 0.03 \\
Complete & $19(32.2)$ & $6(20.0)$ & $13(44.8)$ & \\
Partial & $4(6.8)$ & $1(3.3)$ & $3(10.3)$ & \\
Non-lifting & & & & \\
Histology, $\mathrm{n}(\%)$ & $29(49.2)$ & $15(50.0)$ & $14(48.3)$ & 0.33 \\
Tubular adenoma & $18(30.5)$ & $7(23.3)$ & $11(37.9)$ & \\
Sessile serrated adenoma & $12(20.3)$ & $8(26.7)$ & $4(13.8)$ & \\
TA+ HGD & & & & 0.71 \\
\hline Piecemeal resection, $\mathrm{n}(\%)$ & $42(71.2)$ & $22(73.3)$ & $20(69.0)$ & 0.78 \\
\hline En bloc resection, $\mathrm{n}$ (\%) & $17(28.8)$ & $8(26.7)$ & $9(31.0)$ & 0.01 \\
\hline Recurrence at surveillance, $\mathrm{n}(\%)$ & $6(10.2)$ & $0(0.0)$ & $6(20.7)$ & \\
\hline
\end{tabular}

\section{Secondary Outcome}

Major adverse events were observed in a total of 6 patients (12.5\%), all being post-polypectomy bleeding. Of these, post-polypectomy bleeding occurred in 2 patients (8.0\%) in the hAPC-assisted EMR group and 4 patients $(17.4 \%)$ in the standard EMR group $(p=0.41)$. Despite there being no statistically significant difference between the two groups, there was a trend toward less post-procedural adverse events with the use of hAPC-assisted EMR compared to standard EMR.

\section{Conclusions}

Hybrid APC assisted EMR decreases the rate of local recurrence for laterally spreading lesions and large colon polyps compared to standard EMR. In addition, it may lead to a reduced rate of delayed post-EMR bleeding.

\section{Abbreviations}

EMR: endoscopic mucosal resection

LSL: laterally spreading lesion

APC: argon plasma coagulation 
hAPC: hybrid argon plasma coagulation

STSC: snare tip soft coagulation

\section{Declarations}

Ethics approval and consent to participate- retrospectively registered. This study was approved by the Penn State Milton S. Hershey Medical Center Institutional Review Board (IRB \#12499). All participants provided informed consent for the procedure.

Consent for publication- not applicable

Availability of data and materials- The datasets used and/or analysed during the current study are available from the corresponding author on reasonable request.

Competing interests- The authors declare that they have no competing interests.

Funding- not applicable

Authors' contributions- $\mathrm{AG}$ and $\mathrm{CL}$ analyzed and interpreted the patient data regarding all EMRs during the study period. JL performed all of the procedures in the data set. All authors read and approved the final manuscript.

Acknowledgements- Not applicable.

\section{References}

1. Rotondano G, Bianco MA, Buffoli F, et al. The Cooperative Italian FLIN Study Group: prevalence and clinico-pathological features of colorectal laterally spreading tumors. Endoscopy. 2011;43:856-61.

2. Kandel P, Wallace MB. Colorectal endoscopic mucosal resection (EMR). Best Pract Res Clin Gastroenterol. 2017;31:455-71.

3. Raju G, Lum P, et al. Outcome of Endoscopic Mucosal Resection As an Alternative to Surgery in Patients with Complex Colon Polyps. Gastrointest Endosc. 2016;84:315-25.

4. Law R, Das A, Gregory D, et al. Endoscopic Resection is Cost-effective Compared to Laparoscopic Resection in the Management of Complex Colon Polyps: An Economic Analysis. Gastrointest Endosc. 2016;83:1248-57.

5. Ahlenstiel G, Hourigan LF, Brown G, et al. Actual endoscopic versus predicted surgical mortality for treatment of advanced musocal neoplasia of the colon. Gastrointest Endosc. 2014;80:668-76.

6. Rosenberg N. Submucosal saline wheal as safety factor in fulguration or rectal and sigmoidal polypi. AMA Arch Surg. 1955;70:120-2.

7. Waye JD, Rex DK, Williams CB. Colonoscopy: Principles and Practice, Second Edition, 2009. 
8. Shirai M, Nakamura T, Matsuura A, et al. Safer colonoscopic polypectomy with local submucosal injection of hypertonic saline-epinephrine solution. Am J Gastroenterol. 1994;89:334-8.

9. Klein A, Bourke MJ. Advanced polypectomy and resection techniques. Gastrointest Endosc Clin N Am. 2015;25:303-33.

10. Sethi A, Song L. Adverse Events Related to Colonic Endoscopic Mucosal Resection and Polypectomy. Gastrointest Endosc Clin N Am. 2015;25:55-69.

11. Moss A, Williams SJ, Hourigan LF, et al. Long-term adenoma recurrence following wide-field endoscopic mucosal resection (WF-EMR) for advanced colonic mucosal neoplasia is infrequent: results and risk factors in 1000 cases from the Australian Colonic EMR (ACE) study. Gut. 2015;64:57-65.

12. Buchner AM, Guarner-Argente C, Ginsberg GG. Outcomes of EMR of defiant colorectal lesions directed to an endoscopy referral center. Gastrointest Endosc. 2012;76:255-63.

13. Kaltenbach T, Anderson J, et al. Endoscopic Removal of Colorectal Lesions: Recommendations by the US Multi-Society Task Force on Colorectal Cancer. Am J of Gastroenterol. 2020;115:435-64.

14. Yang D, Othman M, et al. Endoscopic Mucosal Resection vs Endoscopic Submuocsal Dissection For Barrett's Esophagus and Colorectal Neoplasia. Clin Gastroenterol Hepatol. 2019;17:1019-28.

15. Ortiz A, Bhargavi P, et al. Endoscopic Mucosal Resection Recurrence Rate for Colorectal Lesions. South Med J. 2014;107:615-21.

16. Mannath J, Subramanian V, et al. Polyp Recurrence After Endoscopic Mucosal Resection of Sessile and Flat Colonic Adenomas. Dig Dis Sci. 2011;56:2389-95.

17. Holmes I, Kim HG, Yang DH, et al. Avulsion is superior to argon plasma coagulation for treatment of visible residual neoplasia during EMR of colorectal polyps (with videos). Gastrointest Endosc. 2016;84:822-9.

18. Kumar V, Broadley H, Rex DK. Safety and efficacy of hot avulsion as an adjunct to EMR (with videos). Gastrointest Endosc. 2019;89:999-1004.

19. Bahin FF, Pellise M, Williams SJ, et al. Extended endoscopic mucosal resection does not reduce recurrence compared with standard endoscopic mucosal resection of large laterally spreading colorectal lesions. Gastrointest Endosc. 2016;84:997-1006.e1.

20. Klein A, Tate DJ, Jayasekeran V, et al. Thermal Ablation of Mucosal Defect Margins Reduces Adenoma Recurrence After Colonic Endoscopic Mucosal Resection. Gastroenterol. 2019;156:60413.e3.

21. Kandel P, Werlang M, et al. Prophylactic Snare Tip Soft Coagulation and Its Impact on Adenoma Recurrence After Colonic Endoscopic Mucosal Resection. Dig Dis Sci. 2019;64:3300-6.

22. Rastogi A, Keighley J, Singh V, et al. High accuracy of narrow band imaging without magnification for the real-time characterization of polyp histology and its comparison with high-definition white light colonoscopy: a prospective study. Am J Gastroenterol 2009; 104: 2422-2430.. 
23. Participants PW. The Paris endoscopic classification of superficial neoplastic lesions: esophagus, stomach, and colon: Nov 30 to Dec 1, 2002. Gastrointest Endosc 2003; 58: 3-43..

24. Pohl H, Grimm I, et al Clip Closure Prevents Bleeding After Endoscopic Resection of Large Colon Polyps in a Randomized Trial. Gastroenterol 2019; 157: 977-984.

25. ASGE Standard of Practice Committee. Management of antithrombotic agents for endoscopic procedures. Gastrointest Endosc. 2009;70:1060-70., Anderson, M.A., Ben-Menachem, T., et al.

26. ASGE Standard Practive Committee. Antibiotic prophylaxis for $\mathrm{Gl}$ endoscopy. Gastrointest Endosc. 2015;81:81-9., Khashab, M.A., Chithadi, K.V., et al.

\section{Figures}

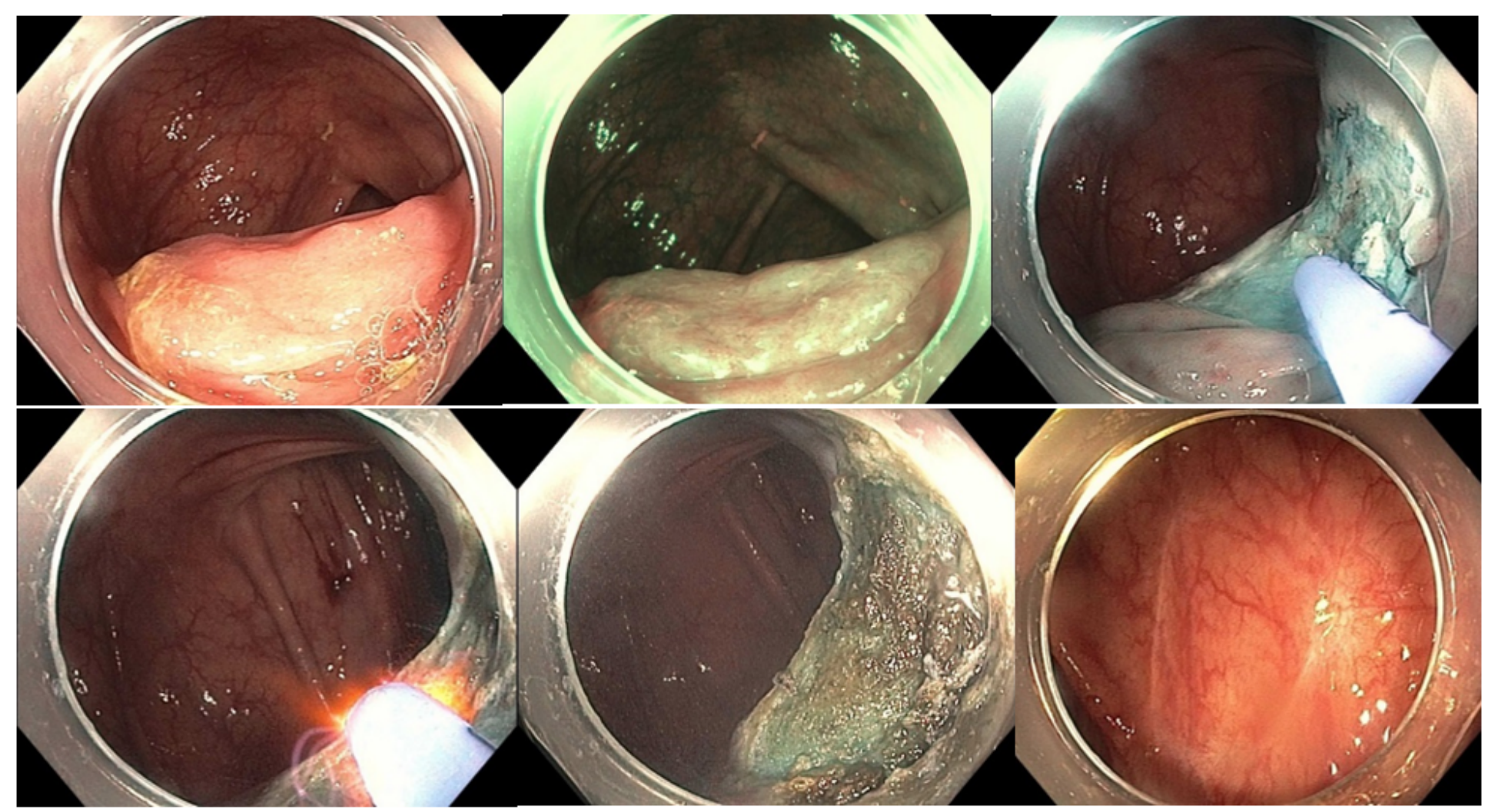

\section{Figure 1}

Resection of a 25mm sessile serrated polyp using hAPC assisted EMR: a) Polyp with white light and b) under NBI, c) Post resection and relifting the base, d) ablating the base, e) post-ablation, f) scar at follow up 